\title{
Facultad por Facultad. Origen familiar y empleo de los licenciados en CCSS y Humanidades de la UCM en el año $2003^{1}$
}

\section{Faculty by Faculty. Family Background and Employment among Social Sciences and Humanities Graduates of the UCM in 2003}

\author{
Julio CARABAÑA MORALES y Gloria de la FUENTE BLANCO» \\ Universidad Complutense de Madrid
}

Recibido: Noviembre 2014

Evaluado: Enero 2015

Aceptado: Enero 2015

\section{Resumen}

Muchos estudios encuentran un efecto del origen social sobre la ocupación y el salario incluso tras controlar la educación. Este efecto, que suele ser pequeño, puede ser un artificio resultante del deficiente control de la educación. Este trabajo examina la importancia de controlar en detalle la educación desagregando las carreras universitarias. Estudiamos el clasismo del mercado de trabajo para una promoción de titulados en Ciencias Sociales y Humanidades de la Universidad Complutense de Madrid (UCM) en los seis años entre su graduación en 1997 y 2003. Esta es la fecha de la encuesta gracias a la cual podemos medir la influencia del status social de los padres sobre las oportunidades de empleo de los hijos no con título universitario en general, sino con el mismo título universitario. Encontramos que la influencia del origen social sobre la clase profesional y los ingresos disminuye mucho cuando se controlan las titulaciones, y que no se observa en la mayor parte de ellas, pero sí en algunas, en concreto Políticas y Sociología y Económicas. Esta concreción allana el camino para investigar las vías por las que esta influencia se produce.

Palabras clave: origen social, facultades universitarias, clasismo, mercado de trabajo, movilidad social, educación e ingresos.

\footnotetext{
${ }^{1}$ Una primera versión de este estudio fue presentada en la II Reunión entre congresos del CIES (Comité de Investigación de Estratificación Social) de la Federación Española de Sociología, que tuvo lugar en la UNED en 2005. Luego quedó sin terminar por, entre otras razones, la enfermedad de Gloria de la Fuente. Julio Carabaña ha completado la presente versión con motivo del homenaje póstumo ofrecido a Gloria en la Facultad de Educación de la UCM en Junio de 2013. Miguel Caínzos, que organizó la reunión del CIES de 2005, ha hecho observaciones a las sucesivas redacciones del trabajo que lo han mejorado notablemente. Lo mismo ha ocurrido con las de Luis Ortiz, Fabrizio Bernardi y Sandra Fachelli, a la versión anterior a esta.
} 


\begin{abstract}
Many studies find an effect of social origins on occupation and income, even after controlling education. This effect, usually rather small, might be an artifice resulting from poor control of education. The present paper examines the importance of thoroughly controlling education taking into account singular university degrees. We study the 'classism' of the labour market for a cohort of Graduates in Social Sciences and Humanities from the University Complutense at Madrid (UCM) in the six years from their graduation in 1997 until 2003. This is the year of the survey through which we can measure the influence of the social status of the parents on the working opportunities of their children, not only with a university degree, but with the same university degree. We find that the effect of social background on the professional class and income wanes when taking diplomas into account, and does not affects the majority of the professional occupations, but some of them, such as Political Sciences, Sociology and Economics. This finding paves the way to test the ways through which this influence operates.
\end{abstract}

Keywords: social background, university faculties, classism, labor market, social mobility, education and income.

¿Vale lo mismo un título académico para todo el mundo, o su valor depende de ciertas características adscritas de los individuos, como su origen social? Quizás sea exagerado decir que se trata de una cuestión de actualidad política. Han dejado de ser moneda corriente en España los 'padrinos', 'enchufes' y 'recomendaciones', y lo que no hace mucho se consideraba un vicio nacional, ahora aparece en forma de casos singularizados y socialmente inadmisibles. Pero no por ello deja de ser menor su interés sociológico, directamente ligado a la discusión sobre la sustitución del particularismo por el universalismo en los procesos de modernización, tal y como la formulara Parsons (1964). O ligado, en otros términos, al debate sobre la meritocracia, que exige, en palabras de Goldthorpe, que "la asociación entre el logro educativo y el nivel de empleo se torne similar para individuos de diferentes orígenes sociales" (Goldthorpe y Jackson, 2005). Lo que aquí se estudia es una parte de la cuestión, a saber, si la contratación de licenciados está influida por su clase social de origen, o, dicho de otro modo, si hay 'clasismo' en el mercado de trabajo de los licenciados. Por insuficiencia de casos en la muestra, el estudio no incluye la adscripción en sentido estricto, es decir, la transmisión por 'herencia' del trabajo por cuenta propia.

La multitud de estudios empíricos realizados hasta la fecha ha encontrado una influencia nula o muy pequeña del origen social de las personas sobre su ocupación y sus ingresos una vez controlados sus estudios (efectos directos). Estos estudios provienen de diversas corrientes de investigación: la tradición del logro de estatus y la movilidad ocupacional, la escuela del capital humano y el estudio de la sobreeeducación.

La influencia directa del origen social en el logro de estatus, calculada primero por Blau y Duncan, puede resumirse en un coeficiente beta (o tipificado) en torno a 0,18 , equivalente al 3\% de la desigualdad total (Blau y Duncan, 1967:169-70). El mismo cálculo para Inglaterra algunos años después dio un resultado de 0,20 , equivalente a un 4\% de la desigualdad total (Halsey y Ridge, 1977:178). Las teorías de la 
modernización son congruentes con una disminución entre los más jóvenes, encontrada en Estados Unidos (Featherman y Hauser, 1978) e Inglaterra (Halsey y Ridge, 1977), y también son coherentes con el hallazgo más reciente de influencias todavía menores, $1,7 \%$ de la varianza total en Estados Unidos (Guzzo 2002). Ahora bien, en Inglaterra Jackson, Goldthorpe y Mills (2008) no encuentran disminución de los efectos con el tiempo, y Bukodi y Goldthorpe (2011) no la encuentran entre los nacidos en 1946, aunque sí entre los nacidos en 1958 y 1970. Controlando incluso las capacidades cognitivas, estos autores encuentran que los hijos de profesionales medios y no manuales (clases II y III) superan en unos diez puntos a los hijos de pequeños empresarios y obreros (clases IV a VII) en el acceso a las clases I-II (profesionales y técnicos), y que los hijos de profesionales y directivos altos (clase I) los superan en 15 puntos. En la misma línea se mueven los resultados de Breen para Irlanda del Norte (Breen, 2003). Las estimaciones llevadas a cabo en España han dado por lo común efectos mayores: 0,25 con datos de una muestra de padres de alumnos en Madrid y Guadalajara (Carabaña, 1983:107); 0,26 con una muestra nacional del CIS (Rodríguez Menés, 1993:105); con otra encuesta nacional, la ECBC (Carabaña y otros, 1992) Echevarría (2009) encontró 0,25, equivalentes al $6 \%$ de la desigualdad total en el estatus de las ocupaciones. Con los datos, superiores, de la Encuesta Sociodemográfica (ESD, INE, 1991) se encuentra que entre los jóvenes la influencia directa del origen social se queda en un coeficiente beta de 0,14 , equivalente al $2 \%$ de la varianza, menor que en las generaciones de más edad (Carabaña, 1999:353). Con los mismos datos y otros más recientes (en particular el estudio CIS 2634 de 2006), Bernardi ha encontrado probabilidades $13 \%$ mayores de acceso a profesionales entre los hijos e hijas de profesionales, y de $23 \%$ limitándose a los hombres de 28 a 45 años (2012, tabla 2a).

Limitando la atención a la enseñanza universitaria, como nosotros haremos aquí, los efectos del origen social se hacen todavía más pequeños, como sería de esperar. En Estados Unidos, Hout encontró que la graduación en un college cancelaba el efecto del estatus de origen en los ochenta (Hout, 1988), un resultado confirmado recientemente por Torche (2011) para los noventa. Pero Torche encontró además que el status familiar vuelve a pesar en los títulos superiores al college, los de las Graduate Schools como Medicina o Derecho que equivalen más directamente a las licenciaturas españolas. En particular, los hombres originarios del tercio de hogares con ingresos más bajos ganan, aún con títulos iguales, hasta $60 \%$ menos que los procedentes del tercio de hogares con ingresos más altos (Torche, 2011). En algunos países europeos también se encuentran efectos del estatus familiar en el trabajo de los universitarios. Opheim (2007) encontró en Noruega un efecto de $1 \%$ de la varianza (o beta de 0,10 ), que evalúa como pequeño. La diferencia que encuentran Jackson, Goldthorpe y Mills en Inglaterra es asimismo de un par de puntos porcentuales (Jackson y Goldthorpe y Mills, 2008). En España, se han encontrado hasta ahora efectos pequeños del estatus familiar en el empleo de los universitarios. Con la Encuesta Sociodemográfica (INE, 1993), que llega hasta 1990 se ha encontrado una influencia pequeña y estable en el tiempo de la clase del padre en la probabilidad de subempleo (Carabaña, 2000:560 ss). Entre los licenciados que entraron a trabajar entre 1967 y 1976, los hijos de profesionales tenían profesiones cuyo prestigio estaba diez puntos sobre la media y más de veinte puntos por encima de 
los licenciados hijos de gente del campo; y entre 1982 y 1991 empeoraron los empleos de todos los titulados que no eran hijos de profesionales (Carabaña, 1996). Limitando la observación a las profesiones para cuyo ejercicio se exige título universitario, la proporción media de acceso estuvo hasta 1991 en torno al 60\%, teniendo los hijos de profesionales liberales una ventaja de 10 puntos sobre los demás (Carabaña, 2004). Considerando únicamente universitarios, pero de todas las edades, Bernardi obtiene la misma ventaja del $13 \%$ en el acceso a la clase EGPI que para todos los niveles de estudio (Bernardi, 2012: tabla 5). Con los datos de la encuesta PIACC de la OCDE sobre competencias de adultos, Martínez García ha encontrado que entre la población universitaria de 25 a 64 años las tasas de sobrecualificación objetiva y subjetiva son diez puntos mayores entre quienes tienen padres con estudios primarios que entre quienes tienen padres con estudios universitarios (Martínez García, 2014:128). En los últimos años, los efectos parecen haberse desvanecido, al menos entre los egresados de las universidades catalanas en 2004 (Fachelli y Planas, 2011) y 2008 (Fachelli y Planas, 2014).

La influencia del status de origen sobre los resultados laborales puede deberse a factores muy diversos, que no son sólo de tipo particularista o adscriptivo, sino también de tipo universalista. Entre estos, el control de las capacidades individuales es clásico entre economistas (Psacharopoulos, 1975) y sociólogos (Duncan y otros, 1972; Jencks y otros, 1972; Sewell y Hauser, 1975). El factor más obvio es la diversidad de los estudios universitarios en duración y especialidades, que se traduce en fuertes diferencias en sus salidas laborales, abundantemente estudiadas en España (García de Cortázar, 1987; Lamo de Espinosa, 1988; recientemente Iriondo y otros, 2009, Barone y Ortiz, 2010). Ahora bien, muchos estudios, al menos desde Bourdieu y Passeron (1964), han señalado grandes diferencias en la composición social de los diversos ciclos y carreras (recientemente v.gr. Berggren, 2008; Gerber y Cheung, 2008; en España, Latiesa, 2000; Ariño y Llopis, 2011; Ustrell, 2011). Resulta por tanto bastante obvio preguntarse hasta qué punto el control de estas diferencias internas a la Universidad, un factor universalista, da cuenta de la relación observada entre origen social y resultados laborales. Según Torche (2011), los titulados en Graduate Schools de familias más ricas ganan más en parte porque están sobrerepresentados en los títulos más lucrativos (negocios, derecho o medicina), pero también en parte porque ganan más con el mismo título. Calmand y Epiphane (2010) encontraron que aun controlando el nivel de los estudios universitarios (cuya jerarquía es en Francia muy acusada) y con una aproximación muy general a la rama, los hijos de cuadros tienen más probabilidades de ser cuadros a los tres años de terminar los estudios que los demás. Triventi (2013) con datos del estudio internacional REFLEX distingue grandes ramas de estudio (Humanidades, Ciencias Sociales, Técnicas, Ciencias Naturales, Salud) y encuentra que las diferencias entre ellas en prestigio e ingresos dan cuenta de todo el efecto de los estudios de los padres sobre esas mismas variables en Noruega, y en una gran parte en Italia y España (más precisamente, en unos dos tercios). En su tesis doctoral, Navarro Cendejas analizó las universidades catalanas tomando como variable dependiente la pertenencia al grupo, formado mediante análisis de correspondencias, con mejor situación en términos de tiempo de empleo, tipo de contrato, ingresos y adecuación entre estudios y trabajo. El origen social pierde todo efecto sobre esta 
variable una vez que se controlan los niveles (corto-largo) y las ramas de estudio, que son Humanidades, Sociales, Derecho y Administración de Empresas, Experimentales, Salud e Ingenierías (Navarro, 2013:271). En cambio, Bernardi encuentra que el control de 83 campos de estudio tan sólo reduce en un punto la ventaja del 13\% en el acceso a la clase EGPI de los hijos de padres de esa misma clase (2012, tabla 7).

\section{Hipótesis, datos y métodos}

Lo anterior no pretende ser una revisión exhaustiva de la literatura, sino tan solo servir para enmarcar el alcance del presente estudio, cuya contribución quiere ser doble. De una parte, pretendemos estudiar precisamente a los titulados de la UCM en un momento de auge del ciclo económico. ¿Podemos extender a los titulados de la UCM en 2003 no ya lo encontrado en Estados Unidos o Inglaterra, sino en Cataluña o en el resto de España? La fecha de 2003 adquiere particular relevancia con la crisis actual, pues proporciona una referencia precisa para cuando se estudien sus efectos. De otra parte, pretendemos ir más allá de las investigaciones precedentes diferenciando carreras singulares, o por lo menos grupos de de la misma Facultad. . Esta posibilidad de captar la heterogeneidad de las carreras es el principal valor añadido por nuestro estudio a la investigación de la cuestión en general.

Nuestra hipótesis central es la que cabría esperar de lo visto, a saber, que la heterogeneidad no observada de los títulos infla la correlación del origen social con las condiciones de empleo. Si en los títulos con mejores salidas hay más alumnos de origen social alto, distinguiendo entre títulos se sitúa la influencia del origen social en su lugar propio, que es la elección de carrera, no posteriormente en el mercado de trabajo. Esperamos, por tanto, que la influencia del origen social sobre las condiciones de trabajo de los titulados, que ya en el conjunto de los títulos no debería ser muy grande, se reduzca mucho, o se elimine del todo, cuando la calculemos título por título.

Podemos contrastar esta hipótesis gracias a una encuesta a Titulados de Ciencias Sociales y Humanidades de la UCM (a la que llamamos UCM03) cuyo propósito inicial era averiguar los efectos sobre la inserción profesional de los cambios en los planes de estudio de los años 90. La mayor parte de los encuestados terminaron la carrera en 1997 y fueron entrevistados 5 años después, en 2003. La encuesta incluía también diplomados, que no se consideran en este estudio, centrado en las licenciaturas ${ }^{2}$. El diseño muestral se llevó a cabo de modo que se obtuvieran muestras representativas de cada titulación, a fin de poder observar la heterogeneidad entre los títulos y no se confundiera con otros factores. Las entrevistas que se pudieron financiar con una ayuda de la CICYT se distribuyeron entre las titulaciones a estudiar de modo

\footnotetext{
${ }^{2}$ La encuesta era el núcleo del Proyecto de Investigación "Nuevas titulaciones y mercado de trabajo. Factores académicos y sociales que condicionan la inserción laboral de los universitarios con titulaciones no tecnológicas", dirigido por Gloria de la Fuente, que fue financiado por el programa SEC 2001-14-17 del MECD. El cuestionario CEFAL (eventos familiares, académicos y laborales) se pasó por CATI durante la primavera de 2013 por la empresa IMOP.
} 
que se minimizara un error igual en cada una de ellas. En total se entrevistaron 2272 egresados, de los cuales 706 fueron diplomados y 1566 licenciados. La tabla I reproduce la distribución de estos por licenciaturas con sus tamaños de población y muestras, más los errores típicos de cada una en el supuesto de distribución 50\%/50\%.

\begin{tabular}{lcccc}
\hline \multicolumn{1}{c}{ Carrera } & N & n & $\begin{array}{c}\text { Error } \\
\text { típico }\end{array}$ & I de C 95\% \\
\hline Económicas & 876 & 144 & 3,48 & 6,96 \\
\hline Empresariales & 1599 & 147 & 3,74 & 7,48 \\
\hline A. y D. Empresas & 603 & 119 & 3,67 & 7,34 \\
\hline Periodismo & 973 & 111 & 4,2 & 8,4 \\
\hline Publicidad & 450 & 82 & 4,52 & 9,02 \\
\hline Imagen & 303 & 56 & 5,44 & 10,88 \\
\hline CC Políticas & 596 & 68 & 5,37 & 10,74 \\
\hline Sociología & 513 & 63 & 5,52 & 10,04 \\
\hline CC. Educación & 485 & 60 & 5,66 & 11,3 \\
\hline Pedagogía & 313 & 53 & 5,7 & 11,4 \\
\hline Psicopedagogía & 170 & 50 & 4,99 & 8,98 \\
\hline Psicología & 609 & 114 & 3,8 & 7,6 \\
\hline Derecho & 2774 & 157 & 3,76 & 7,52 \\
\hline Filo. Hispánica & 227 & 49 & 5,6 & 11,2 \\
\hline Filo. Inglesa & 345 & 53 & 5,81 & 11,62 \\
\hline Filosofía & 128 & 44 & 4,95 & 9,88 \\
\hline Geo e Historia & 760 & 78 & 5,08 & 10,16 \\
\hline Geografía & 53 & 24 & 5,58 & 11,16 \\
\hline Historia & 159 & 46 & 5,24 & 10,46 \\
\hline Historia Arte & 172 & 48 & 5,2 & 10,4 \\
\hline Total & 12108 & 1566 & &
\end{tabular}

Tabla I. Tamaño y error muestral por titulaciones

Fuente: elaboración de la Encuesta UCM93

Por desgracia la muestra de cada título académico es demasiado pequeña para los análisis multivariados. El modo más inocuo de agrandarla es agrupar los títulos en Facultades. Hacemos los siguientes grupos:

- Empresariales. Comprende los títulos de Económicas (a), Empresariales y Administración de Empresas.

- Derecho: un solo título.

- Comunicación: Comprende Periodismo, Publicidad e Imagen.

- Letras: comprende Filosofía, Geografía e Historia (a), Geografía, Historia, Historia del Arte, Filología Española y Filología Inglesa. 
- Sociales: comprende Ciencias Políticas y Sociología.

- Psicología: Un solo título.

- Pedagogía: comprende Ciencias de la Educación (a), Pedagogía y Psicopedagogía.

De estos títulos, los señalados con (a) fueron sustituidos por los otros tras las reformas de los planes de estudio que tuvieron lugar durante los noventa, consecuencia de la LRU. De ahí vienen ciertas diferencias en el año de terminación de los estudios, que no son de relevancia aquí.

Los títulos de Letras se agrupan en una sola Facultad porque todos estaban en la antigua Facultad de Filosofía y Letras, que se diversificó luego en las de Filología e Historia. En relación al mercado de trabajo, siguen teniendo en común que su salida principal es la enseñanza.

El análisis exploratorio de los datos llevó a agrupar los valores de algunas variables para adaptarlos al análisis y simplificar la exposición.

Estudios de los progenitores. Los estudios de las madres añaden muy poca información a los estudios de los padres. Estos se reducen a cuatro niveles para las ecuaciones lineales (primarios, medios, diplomados y licenciados) y a tres (primarios, medios y universitarios) para las logísticas ${ }^{3}$.

Ocupación de los progenitores. La ocupación de las madres falta en muchos casos y cuando la hay es redundante con la de sus cónyuges. Las ocupaciones de los padres se agruparon de acuerdo con el esquema EGP y con los simples grandes grupos de la CNO. En ambas clasificaciones el análisis exploratorio no muestra diferencias sistemáticas más que entre los padres profesionales y el resto, así que utilizamos en las regresiones la variable EGPPI, que distingue entre padres profesionales y directivos (I) y el resto.

Calificaciones académicas. Una tercera parte de los entrevistados no dio respuesta a la pregunta por la nota media de su carrera. Comprobado que no había diferencias en las demás variables entre los que no contestaron y los que simplemente aprobaron, se construyó la variable 'NOTABLE' con valor 0 por defecto y valor 1 para los que tuvieran esta calificación o sobresaliente (muy pocos).

Del objeto de estudio, el trabajo, se tomaron las dos características más importantes, la ocupación y los ingresos. En conjunto, había un 5\% de inactivos y un 5\% de parados, pero como tomamos la ocupación actual o última solo quedaron sin ocupación 21 entrevistados que no habían trabajado nunca. El último trabajo lo hacen por cuenta propia el $8,2 \%$ de los entrevistados, siendo las carreras con más empleados por cuenta propia Derecho (21\%) e Imagen (16\%). La ocupación se trató del mismo modo que la de los padres, quedándonos finalmente con la separación entre ocupaciones de

\footnotetext{
${ }^{3}$ La diferencia entre diplomado y licenciado influye sobre la correlación, pero produce una variable dummy redundante.
} 
profesionales y directivos (EGPI) y el resto. Los ingresos se habían precodificado en ocho intervalos en el cuestionario, y así se han tratado en todos los análisis estadísticos.

El método seguido ha consistido en calcular la influencia de la clase del padre en la del hijo controlando el sexo de los alumnos y su nota media en la carrera. En realidad, el sexo se incluye con fines comparativos, pues su relación con la clase de origen (hay más universitarias que universitarios de clases obreras) es muy tenue. Más plausible es la presunción de que la influencia de la clase se debe en realidad a diferencias académicas ${ }^{4}$, pero la desmiente la correlación entre ambas; así que también la nota media tiene en realidad utilidad comparativa. Para contrastar la hipótesis del efecto de la carrera, la estimación de este sencillo modelo se realiza primero para el conjunto de las carreras y luego para cada grupo de ellas por separado.

\section{Resultados sobre profesiones}

La tabla II contiene los estadísticos descriptivos de las principales variables por Facultades. La feminización de las carreras varía entre el aproximadamente 50\% de mujeres en Económicas y Derecho, el 60\% en Comunicación, Letras y Sociales y el $70 \%$ de Psicología y Pedagogía. Económicas y Derecho se distinguen también por tener los estudiantes con origen social más alto: aproximadamente la mitad tienen padres universitarios o profesionales. En las demás Facultades los porcentajes disminuyen notablemente, hasta el entorno del $20 \%$. El porcentaje de alumnos cuya nota media es de notable o más está en torno al $30 \%$ en todas las Facultades excepto en Económicas, con el 17\%, y Psicología, con el 12\%. A la cabeza de empleados en ocupaciones pertenecientes a la clase EGPI (profesionales y directivos) está Económicas, con más del 50\%, seguida de cerca por Derecho y Psicología. El resto de titulaciones está en torno al $25 \%$.

\footnotetext{
${ }^{4}$ No ha sido confirmada recientemente ni por Bukodi y Goldthorpe (2011) ni por Bernardi (2012). Nótese que ambos autores utilizan indicadores de capacidad general de los individuos, mientras que las calificaciones en la carrera que aquí usamos pueden considerarse indicadores directos de la capacidad profesional, con más probabilidades de influir en los resultados laborales.
} 


\begin{tabular}{lrrrrrr}
\hline FACULTAD & \multicolumn{1}{l}{$\begin{array}{l}\text { Sexo } \\
\text { femenino }\end{array}$} & $\begin{array}{l}\text { Padre } \\
\text { Universitario }\end{array}$ & $\begin{array}{l}\text { Padre } \\
\text { EGP I }\end{array}$ & $\begin{array}{l}\text { Nota } \\
\text { Notable }\end{array}$ & $\begin{array}{l}\text { Empleo } \\
\text { EGP I }\end{array}$ & \multicolumn{2}{l}{ Total } \\
\hline Empresariales & 194 & 193 & 164 & 65 & 207 & 379 \\
\hline & $51,1 \%$ & $51,9 \%$ & $43,2 \%$ & $17,1 \%$ & $54,6 \%$ & $100,0 \%$ \\
\hline Derecho & 177 & 194 & 161 & 109 & 146 & 342 \\
\hline & $51,6 \%$ & $59,3 \%$ & $47,1 \%$ & $31,9 \%$ & $42,7 \%$ & $100,0 \%$ \\
\hline Comunicación & 128 & 83 & 51 & 63 & 39 & 212 \\
\hline Humanidades & $60,4 \%$ & $39,7 \%$ & $23,9 \%$ & $29,6 \%$ & $18,4 \%$ & $100,0 \%$ \\
\hline & 167 & 72 & 61 & 94 & 61 & 300 \\
Sociales & $55,7 \%$ & $24,8 \%$ & $20,3 \%$ & $31,3 \%$ & $20,3 \%$ & $100,0 \%$ \\
\hline & 76 & 33 & 30 & 37 & 39 & 136 \\
Psicología & $55,5 \%$ & $24,6 \%$ & $22,1 \%$ & $27,0 \%$ & $28,7 \%$ & $100,0 \%$ \\
\hline & 55 & 16 & 21 & 9 & 35 & 75 \\
Pedagogía & $73,3 \%$ & $21,3 \%$ & $28,0 \%$ & $11,8 \%$ & $46,7 \%$ & $100,0 \%$ \\
\hline & 75 & 24 & 17 & 45 & 27 & 119 \\
Total & $63,0 \%$ & $20,7 \%$ & $14,3 \%$ & $37,8 \%$ & $22,7 \%$ & $100,0 \%$ \\
\hline
\end{tabular}

Tabla II. Características principales de los alumnos por Facultades

Fuente: elaboración de la Encuesta UCM93

Como puede apreciarse, las Facultades con mejores salidas profesionales tienen más estudiantes varones e hijos de padres profesionales. Esta coincidencia apunta en la dirección de la hipótesis principal, a saber, que cuando no se desagrega por Facultades se atribuyen a la clase social y al sexo ventajas en el mercado de trabajo que en realidad provienen de los estudios cursados. Los análisis multivariados de las tablas siguientes corroboran esta impresión. 
modelo 1

modelo 2

\begin{tabular}{lcccccc}
\hline & B & E.T. & Sig. & B & E.T. & Sig. \\
\hline Sexo masculino &, 101 &, 110 &, 359 &, 043 &, 115 &, 711 \\
\hline $\begin{array}{l}\text { Padre } \\
\text { universitario }\end{array}$ &, 347 &, 147 &, 019 &, 196 &, 157 &, 214 \\
\hline Padre e. medios &, 105 &, 150 &, 486 &,- 002 &, 157 &, 988 \\
\hline Padre EGP I &, 541 &, 135 &, 000 &, 371 &, 142 &, 009 \\
\hline Notable &, 456 &, 121 &, 000 &, 703 &, 130 &, 000 \\
\hline $\begin{array}{l}\text { Empresariales } \\
\text { (ref) }\end{array}$ & & & & & & \\
\hline derecho & & & & $-1,669$ &, 211 &, 000 \\
\hline comunicación & & & & $-1,513$ &, 186 &, 000 \\
\hline humanidades & & & & $-1,022$ &, 222 &, 000 \\
\hline sociales & & & & $-1,444$ &, 257 &, 000 \\
\hline psicología & $-1,129$ &, 114 &, 000 &,- 227 &, 153 &, 138 \\
\hline pedagogía & & & & & & \\
\hline Constante & & & & & & \\
\hline
\end{tabular}

Tabla III. Determinantes del empleo de clase EGP I. Regresión Logística Fuente: elaboración de la Encuesta UCM93

El modelo 1 de la tabla III presenta los resultados de una regresión logística para el conjunto de las carreras, dando a la muestra de cada Facultad el peso de su población en el conjunto. Las variables independientes son el sexo del alumno, los estudios (universitarios y secundarios) y la ocupación (EGP I, profesionales y directivos) de los padres y la nota media de la carreras; la variable dependiente es la probabilidad de tener un trabajo de la clase EGPI. Los resultados pueden resumirse así:

- Hay una diferencia favorable a los varones, aunque no tan grande que sea estadísticamente significativa.

- Dos de loos tres indicadores del origen social -padre con estudios universitarios, padres de clase EGP I- producen coeficientes estadísticamente significativos y sustantivamente importantes.

- Un expediente académico con media de notable o más también mejora las probabilidades de tener un trabajo de clase EGP I; la introducción de esta variable no altera los coeficientes del origen social, con el que no covaría.

Se aprecia mejor la importancia sustantiva de estos coeficientes si se los traduce, aunque sea aproximadamente, a diferencias de porcentajes. Los coeficientes de ca. 0,35 que producen los estudios universitarios reflejan una diferencia de ca. 7 puntos 
porcentuales en relación a los estudios primarios. El coeficiente de 0,54 de tener un padre de la clase EGP I refleja una diferencia de ca. 11 puntos porcentuales en relación al resto de las clases. La referencia es un alumno cuyo padre tiene estudios primarios y no pertenece la clase I, que tiene una probabilidad de ca. $24 \%$ de tener un empleo de clase I. Que el padre tenga más que estudios primarios y sea de la clase EGP I da lugar a una diferencia de 19 puntos porcentuales. Se trata de una diferencia considerable. Su magnitud queda aún mejor ilustrada si la comparamos con la diferencia de 9 puntos porcentuales que produce una calificación media de notable.

El modelo 2 de la tabla III intenta contrastar la hipótesis de que la influencia del origen social tiene lugar a través de la carrera introduciendo en la ecuación cada una de estas con Económicas como referencia. Sus coeficientes reflejan más o menos las diferencias en empleo profesional que ya hemos visto en la tabla II. Los coeficientes de las variables del modelo 1 cambian en direcciones opuestas. El de la variable de logro, la nota media, sube en más de un $50 \%$; el de las variables adscriptivas baja considerablemente, de acuerdo con la hipótesis. Según el modelo 2, el sexo carece totalmente de importancia, y tener un padre con estudios universitarios y empleo profesional da una ventaja de solo 12 puntos en empleo profesional, mientras que un expediente de notable da una ventaja de 15 puntos. Este efecto del control de las Facultades sobre el efecto de las notas es un interesante subproducto del análisis, no previsto cuando lo planteamos.

La tabla III lleva a la conclusión de que esos efectos son comunes a todas las licenciaturas estudiadas, iguales para cada una de ellas. Podemos comprobar si esto es así analizando por separado cada Facultad, que al cabo era la intención con que se diseñó la muestra. En la tabla IV, primero vienen los resultados para el conjunto de las Facultades (el modelo 1 de la tabla III) y a continuación la misma ecuación calculada Facultad por Facultad.

\begin{tabular}{|c|c|c|c|c|c|c|}
\hline & VARÓN & PADRE UNI & $\begin{array}{l}\text { PADRE } \\
\text { SECUND } \\
\end{array}$ & $\begin{array}{l}\text { PADRE } \\
\text { EGPI } \\
\end{array}$ & NOTABLE & $\begin{array}{l}\text { CONS- } \\
\text { TANTE } \\
\end{array}$ \\
\hline TODOS &, $10(, 11)$ &, $35(, 15)^{* *}$ &, $10(, 15)$ &, $54(, 14)^{* * *}$ &, $46(, 12)^{* * *}$ & $-1,13$ \\
\hline EMPRESARIALES &,$- 13(, 21)$ & ,32(,29) & ,30(,29) &, $53(, 25)^{* *}$ &, $71(, 29)^{* * *}$ & $-0,34$ \\
\hline DERECHO &, $09(, 36)$ &, $55(, 51)$ &,$- 40(, 59)$ &,$- 04(, 41)$ & $1,4(, 39)^{* * *}$ & $-1,04$ \\
\hline COMUNICACIÓN &,$- 10(, 33)$ &, $54(, 42)$ &, $39(, 44)$ &, $13(, 40)$ &, $56(, 34)^{*}$ & $-1,85$ \\
\hline HUMANIDADES &, $13(, 27)$ &,$- 23(, 40)$ &, $13(, 33)$ &, $28(, 41)$ &, $27(, 28)$ & $-1,4$ \\
\hline SOCIALES &, $24(, 41)$ &,$- 15(, 61)$ & ,12(,49) & $1,6(, 56)^{* * *}$ &, $108(, 46)$ & $-1,4$ \\
\hline PSICOLOGIA &,$- 16(, 43)$ &, $22(, 57$ &, $11(, 43)$ &, $03(, 49)$ & $1,04(, 65)^{*}$ & $-0,3$ \\
\hline PEDAGOGÍA &, $4(, 4)$ &,$- 18(, 62)$ &, $76(, 45)$ & ,20(,61) &, $29(, 39$ & $-1,7$ \\
\hline
\end{tabular}

Tabla IV. Determinantes del empleo de clase EGP I, por Facultades. Regresión Logística Fuente: elaboración de la Encuesta UCM93 
No sólo vemos ahora que la importancia del origen social disminuye al controlar los títulos, sino también que no se da de modo uniforme en todas las Facultades (en realidad, en los diversos mercados de trabajo de cada título). En ninguna Facultad son estadísticamente significativos los coeficientes de los estudios del padre. Además, el hecho de que presenten tanto signos negativos como positivos asegura de que la falta de significatividad no se debe sólo al tamaño de la muestra, sino sobre todo a su propia falta de sustancia. Sólo en dos Facultades, Empresariales y Sociales, es el coeficiente de que el padre tenga una profesión de clase I EGP significativo y positivo para encontrar una profesión de la misma clase.

Tampoco el aumento de la importancia de las calificaciones académicas que resulta de controlar las Facultades es uniforme en todas ellas. Tener una nota media de notable o más tiene un coeficiente positivo y estadísticamente significativo en tres Facultades, con mucha importancia en Derecho y menos en Empresariales y Comunicación (en Psicología es grande, pero estadísticamente su significatividad no baja de 0,11 ).

El modelo anterior puede dar resultados distorsionados por la colinearidad de las variables de origen social, en particular los estudios superiores y la clase EGP I del padre. La tabla V recoge los resultados de estimar un modelo simplificado, con sólo tres variables, sexo, padre EGPI y notable. Puede apreciarse que son los mismos que con el modelo más complejo.

Para asegurar que la falta de significatividad de los resultados por Facultades no se debe (sólo) a la reducción del tamaño de la muestra, en la parte b de la tabla $\mathrm{V}$ se han calculado además las medias de los coeficientes no significativos. La correspondiente al sexo es más baja que el coeficiente general. La de la clase EGP I del padre es muy inferior al coeficiente general, y no sería significativa con toda la muestra. En cambio, la media de los coeficientes correspondientes a la nota queda muy cercana al coeficiente general. Concretando, en Psicología el coeficiente es grande, y en Humanidades y Pedagogía queda cercano a la media. Juntándolas, puede comprobarse fácilmente que resulta estadísticamente significativo. Es finalmente Sociología la que destaca de las demás Facultades tanto por el alto coeficiente de la profesión paterna como por el coeficiente nulo de las notas. 
A. Regresión logística, modelo simplificado

\begin{tabular}{rcccccccc} 
& \multicolumn{3}{c}{ VARON } & \multicolumn{3}{c}{ PADRE EGP I } & & \multicolumn{3}{c}{ CONSTANTE } \\
\cline { 2 - 8 } & B & E.T. & B & E.T. & B & E.T. & B & E.T. \\
\hline TODOS &, 088 &, 108 &, 713 &, 112 &, 453 &, 118 & $-1,012$ &, 089 \\
EMPRESARIALES &,- 126 &, 202 &, 601 &, 206 &, 687 &, 279 &,- 137 &, 168 \\
DERECHO &, 048 &, 342 &, 266 &, 343 & 1,405 &, 365 &,- 907 &, 314 \\
COMUNICACIÓN &,- 133 &, 331 &, 366 &, 354 &, 566 &, 335 & $-1,609$ &, 260 \\
HUMANIDADES &, 145 &, 264 &, 154 &, 324 &, 314 &, 276 & $-1,452$ &, 206 \\
SOCIALES &, 239 &, 408 & 1,519 &, 448 &, 081 &, 459 & $-1,427$ &, 337 \\
PSICOLOGÍA &,- 146 &, 433 &, 101 &, 429 & 1,018 &, 646 &,- 242 &, 260 \\
PEDAGOGÍA &, 384 &, 382 &, 073 &, 520 &, 448 &, 376 & $-1,546$ &, 311 \\
\hline B. 340
\end{tabular}

B. Media de los coeficientes no significativos

sexo (7) $\quad 0,059$

Padre EGP I (5) $\quad 0,192$

notable (4) $\quad 0,466$

Tabla V. Determinantes del empleo de clase EGPI, por Facultades

Fuente: elaboración de la Encuesta UCM93

\section{Resultados sobre ingresos}

La tabla VIa reproduce las medias de ingresos por Facultades. Por desgracia no están expresados en una unidad con sentido, sino en ocho intervalos arbitrarios. Aun así, se ve claramente que Económicas tiene ingresos más altos y Humanidades y Psicología más bajos que el resto de Facultades, que se sitúan en torno a la cifra (sin sentido) de 5.

La tabla VIb comprueba la importancia de diversos determinantes de los ingresos. Lo primero que hay que advertir es su poco poder explicativo. Cierto que por obra de la pobre unidad de medición la desigualdad no es muy grande (dt. 1,44, coeficiente de variación de 0,30 ). Pero aun así la proporción de varianza que explican las variables es realmente baja, $\%$ en el conjunto. Por Facultades, varía de la nada en Humanidades al $11 \%$ en Ciencias Sociales.

Considerando el conjunto de las Facultades, toda la explicación parece deberse a los factores adscriptivos: ganan más los varones y los hijos de padres con más estudios y con ocupaciones profesionales. El factor de logro, la nota media de la carrera, no parece influir nada.

Facultad por Facultad, la importancia de estos factores adscriptivos disminuye notablemente. En ninguna son estadísticamente significativos los tres. En 
Empresariales lo son el sexo y la profesión paterna; en Derecho sólo el sexo, en Comunicación los estudios paternos, en Sociales la profesión paterna, en Humanidades y Psicología ninguna. En cuanto al factor de logro, , Pedagogía es la única en la que además de la profesión del padre, resulta significativa la nota.

a. Estadísticos descriptivos

\begin{tabular}{lrrr}
\hline & Media & N & Desv. típ. \\
\hline EMPRESARIALES & 5,68 & 368 & 1,23 \\
DERECHO & 5,07 & 283 & 1,48 \\
COMUNICACIÓN & 4,99 & 208 & 1,37 \\
HUMANIDADES & & & \\
SOCIALES & 4,74 & 279 & 1,56 \\
PSICOLOGÍA & 5,05 & 134 & 1,21 \\
PEDAGOGÍA & 4,54 & 72 & 1,59 \\
Total & 4,83 & 116 & 1,47 \\
\hline
\end{tabular}

Tabla VIa. Determinantes de los ingresos, por Facultades

b. Regresión, MCO.

\begin{tabular}{lrrrrr}
\hline & \multicolumn{5}{c}{ PADRE } \\
& \multicolumn{1}{c}{ VARÓN } & PADRE, UNI & \multicolumn{1}{c}{ EGPI } & \multicolumn{1}{c}{ NOTABLE } & CONSTANTE \\
\hline TODOS & $.32(.007)^{* * *}$ & $.069(.033)^{* *}$ & $.37(.09)^{* * *}$ & $.01(.08)$ & 4.6 \\
EMPRESARIALES & $.24(.12)^{* *}$ & $.00(.00)$ & $.57(.15)^{* * *}$ & $.04(.03)$ & 5.3 \\
DERECHO & $.63(.18)^{* *}$ & $.04(.08)$ & $.16(.20)$ & $.10(.18)$ & 4.5 \\
COMUNICACIÓN & $.29(.20)$ & $.18(.08)^{* *}$ & $.006(.25)$ & $.13(.81)$ & 4.5 \\
HUMANIDADES & $-.14(.19)$ & $.04(.08)$ & $.03(.03)$ & $.06(.20)$ & 4.7 \\
SOCIALES & $.27(.20)$ & $.03 .09)$ & $.94(.27)^{* * *}$ & $.22(.23)$ & 4.7 \\
PSICOLOGIA & $.56(.42)$ & $.08(.16)$ & $.05(.45)$ & $.71(.60)$ & 4.6 \\
PEDAGOGÍA & $.37(.26)$ & $.07 .06)$ & $.87(.40)^{* *}$ & $.58(.27)^{* *}$ & 4.5 \\
\hline
\end{tabular}

Tabla VIb. Determinantes de los ingresos, por Facultades 
La tabla VIc refleja además la media de los coeficientes no significativos. La correspondiente al sexo es casi tan grande como el coeficiente para todas las Facultades, lo que implica que su pérdida de significatividad se debe mayormente a la reducción del tamaño muestral. Las correspondientes a los estudios y sobre todo a la profesión paterna, en cambio, disminuyen lo bastante como para justificar la pretensión (la hipótesis principal) de que en el coeficiente de regresión para el conjunto de las Facultades hay un importante efecto de composición. En general, por tanto, podría decirse que los resultados en relación a los ingresos son semejantes, aunque algo menos claros,a los obtenidos respecto a la ocupación en lo que concierne a las variables de origen social; en cambio, en lo que se concierne al sexo, tienen ventaja los varones, mientras que las calificaciones académicas parecen no influir en los ingresos.

\begin{tabular}{lrrrr}
\hline & & & \\
& VARÓN & $\begin{array}{c}\text { PADRE, } \\
\text { UNI }\end{array}$ & \multicolumn{1}{c}{$\begin{array}{c}\text { PADRE } \\
\text { EGPI }\end{array}$} & NOTABLE \\
\hline $\begin{array}{l}\text { c. Media de los coeficientes } \\
\text { no significativos }\end{array}$ & 0,27 & 0,04 & 0,06 & 0,21
\end{tabular}

Tabla VIc. Media de los coeficientes no significativos

\section{Discusión y conclusiones}

Los resultados parecen confirmar, en primer lugar, que en el mercado de trabajo de los licenciados en Ciencias Sociales y Humanidades de la UCM, entre 1997 y 2003 se dio un efecto del origen social sobre la ocupación semejante al encontrado por otros estudios en varios lugares y tiempos, incluida España. Ahora bien, el objetivo de este estudio era comprobar hasta qué punto este efecto es aparente, debido en realidad a que los vástagos de clases más altas eligen carreras con mejores salidas. En general, los resultados confirman nuestra hipótesis de que no tener en cuenta la heterogeneidad de las carreras infla los coeficientes del origen social, con la consecuencia de que se atribuye al mercado de trabajo un efecto particularista que en realidad proviene de la elección de carrera. En general, pues, no puede decirse que el mercado de trabajo haya sido 'clasista' para los licenciados que estudiamos; en concreto, hemos encontrado cierto clasismo en las Facultades de Ciencias Políticas y Sociología y Económicas y Empresariales, y una incierta influencia en los ingresos enComunicación y Pedagogía.

¿Hasta qué punto es generalizable el resultado principal, es decir, qué validez externa le podemos atribuir? Parece altamente improbable que se trate de un fenómeno limitado a las titulaciones, la Universidad o el momento que hemos estudiado, y muy probable que se dé en todas partes. Así lo indica tanto la coincidencia de nuestros resultados con los de otros estudios ya mencionados (Torche, 2011, Triventi, 2013; Navarro, 2013), como el hecho de ser prácticamente universales las condiciones que lo determinan, a saber, diversidad en las salidas laborales de las titulaciones y segregación social a la entrada en ellas. No se ha encontrado hasta ahora ningún lugar donde no se den ambas. 
Secundariamente, hemos encontrado algo parecido respecto al sexo. No se puede calificar el mercado de trabajo de los licenciados en Sociales y Humanidades de sexista si se atiende a la probabilidad de colocarse como profesional, donde no hay diferencias significativas ni siquiera en el conjunto de la muestra. En cambio, es posible hacerlo respecto a los ingresos, donde la diferencia favorable a los varones se mantiene en todas las Facultades menos una, con coeficientes estadísticamente significativos en Empresariales y Derecho. Dado que el trabajo no se planteó con este objetivo, parece prudente dejar el tema sin pronunciarnos sobre la validez externa de estos resultados.

Es también interesante el hallazgo, totalmente imprevisto, de que el control de las carreras aumenta el efecto de las notas sobre la profesión, particularmente en Derecho y Económicas. Yendo facultad por facultad se evita el error de afirmar que la clase de origen es más importante que las notas, y se puede incluso afirmar que ocurre lo contrario. En cuanto a la nula influencia de las notas sobre los ingresos, quizás pueda explicarse porque, a tan solo cinco años de haber terminado los estudios, los alumnos con mejores notas pueden estar todavía en período de aprendizaje, faltando aún para que sobrepasen en ingresos a los de peores notas, que habrían ya dejado de formarse ${ }^{5}$. Aun así, resulta decepcionante (por lo menos para un profesor) que la jerarquía académica de los alumnos tenga tan poco reflejo en los ingresos. En cuanto a la validez externa de este resultado, los elementos de juicio son pocos, así que mejor ser prudentes al respecto. Habrá tiempos y sitios donde las notas cuenten más y otros donde cuenten menos.

Dos facultades se apartan de la pauta de las otras, Sociales y Económicas. En los mercados de trabajo de ambas es muy marcado el efecto de que los padres sean profesionales o directivos, y en el de Políticas y Sociología, además, carecen de importancia las notas. Con los datos de estas carreras se puede continuar la investigación de las vías por las que los licenciados hijos de profesionales tienen más fácil conseguir empleos de profesionales. La vía de las notas es poco prometedora, pues, como acabamos de ver, no tienen correlación con el origen social y su inclusión en la ecuación no altera los coeficientes de las variables paternas. Otras, empero, quedan abiertas. Una es la diferencia entre las carreras en el interior de las Facultades, si bien tiene poco recorrido a la luz de algunos análisis exploratorios. Una segunda es la de la segmentación de los mercados de trabajo. Quizás la peculiaridad de Económicas tenga que ver con rasgos de su mercado de trabajo como su menor vinculación con el sector público.

Concluyendo, el objetivo del estudio era comprobar si no tener en cuenta la diversidad de los títulos académicos lleva a conclusiones exageradas sobre la influencia de la clase social de origen en los mercados de trabajo. El análisis de los datos de la Encuesta UCM03 ha encontrado primero una fuerte influencia del origen familiar en la probabilidad de trabajar en una ocupación de tipo profesional, y también, aunque más débil, en los ingresos. Y luego ha confirmado la hipótesis de que buena

\footnotetext{
${ }^{5}$ Es lo que predice la teoría del capital humano y confirma la investigación. El momento en que debe calcularse la influencia de la escuela sobre los ingresos es el de 'overtaking' de los que dejaron de estudiar antes por los que dejan de estudiar después (cf. Mincer, 1974:16).
} 
parte de esta influencia, si no toda, no es debida al mercado de trabajo, sino a que los hijos de profesionales eligen más las carreras con mejores salidas, como Empresariales y Derecho. Sólo en dos Facultades, Sociales y Empresariales, parece haber algún efecto del status familiar sobre la ocupación y los ingresos, efecto cuyos mecanismos quedan pendientes de investigar.

\section{Referencias bibliográficas}

ARIÑO, A. \& LLOPIS, R. (dirs.) (2011). ¿Universidad sin clases?. Condiciones de vida de los estudiantes universitarios en España (Eurostudent IV), Madrid, Ministerio de Educación.

BARONE, C. \& ORTIZ, L. (2011). Overeducation among European University Graduates:a comparative analysis of its incidence and the importance of higher education differentiation. Higher Education, 61, 325-337.

BERGGREN, C. (2008). Horizontal and Vertical Differentiation within Higher Education-Gender and Class Perspectives. Higher Education Quarterly, 62, 20-39.

BERNARDI, F. (2011). Social origins and inequality in educational returns in the labour market in Spain. Firenze: EUI WP 5/2012.

BLAU, P. \& DUNCAN. O. D. (1967). The American Occupational Structure. New York: John Wiley and Sons.

BREEN, R. (2003). Is Northern Ireland an Educational Meritocracy? Sociology, 37 (4), 657-675.

BOURDIEU, P. \& PASSERON, J.C. (1964). Los estudiantes y la cultura, Barcelona: Labor, 1966.

BUKODI, E.\& J. H. GOLDTHORPE (2011). Social class returns to higher education: chances of access to the professional and managerial salariat for men in three British birth cohorts. Longitudinal and Life Course Studies, 2 (2), 185 - 201.

CARABAÑA, J. (1983). Educación, Ocupación e Ingresos en la España del siglo XX, Madrid: MEC.

CARABAÑA, J.; GONZÁLEZ, J.J.; SERRANO, A.; VALLEJOS, A.; VEREDAS, S.; RIVIERE, J. \& NAVARRO, C. (1992) Encuesta de Estructura, Conciencia y Biografia de clase. Informe Técnico. Madrid: Comunidad de Madrid

CARABAÑA, J. (1996). ¿Se devaluaron los títulos?. REIS, 75, pp.173-213.

CARABAÑA, J. (1999). Dos estudios sobre movilidad intergeneracional en España. Madrid: Argentaria-Visor(dis).

CARABAÑA, J. (2000). Títulos contra paro. ¿Protegen los estudios del desempleo?. Pp. 515-604 en F. Sáez, Formación y empleo, Madrid, Argentaria-Visor.

CARABAÑA, J. (2004). Educación y movilidad social. Pp. 209-239 en Navarro, V, El Estado del Bienestar en España, Madrid: Tecnos. 
DUNCAN, O. D., FEATHERMAN D. L \& B. DUNCAN (1972). Socioeconomic Background and Achievement. New York: Seminar Press.

ECHEVARRÍA ZABALZA, J. (1999). La movilidad social en España, Madrid: Istmo.

FACHELLI, S. \& J. PLANAS. (2011). Equidad y movilidad intergeneracional de los titulados universitarios catalanes. PAPERS Revista de Sociología 96(4), 1307-1331

FACHELLI, S. \& J. PLANAS. (2014). Inserción profesional de los universitarios: de la expansión a la crisis. Revista Española de Sociología, 21, 69-98.

FEATHERMAN, D. L., \& R. M. HAUSER.(1978). Opportunity and Change. New York: Academic Press.

GARCÍA DE CORTÁZAR, M. L. (1987). Educación superior y empleo en España, Madrid: MTSS.

GERBER, T. P. \& CHEUNG, S. Y. (2008). Horizontal Stratification in Postsecondary Education: Forms, Explanations. Annual Review of Sociology, 34, 299-318.

GOLDTHORPE, J. H. \& M. JACKSON (2005). Education-Based Meritocracy:The Barriers to its Realisation. Presentación en la II Reunión entre congresos del CIES (Comité de Investigación de Estratificación Social) de la Federación Española de Sociología. Madrid: UNED.

GUZZO, S. (2002).The Relative Importance of Education: Measuring Societal Inequality in the United States, 1972 to 2000. Paper Presented to ISA, RC28, 2002.

HALSEY, A.H., A. F. HEATH \& J. M. RIDGE (1980). Origins and Destinations: Family, Class and Education in Modern Britain. Oxford: Clarendon Press.

HOUT, M. (1998). More Universalism, Less Structural Mobility. The American Occupational Structure in the 80s. American Journal of Sociology, 93 (6) 13581400.

INE (1993). Encuesta Sociodemográfica 1991. Varios Tomos, Madrid: INE.

IRIONDO, I. (coord.), E. GALLEGO, D. GRANDAL, E.GRACIA, C. DE LA IGLESIA (2009). Inserción Profesional de las principales licenciaturas de la UCM. Informe 2008. Madrid: Ed. Ecobook.

JACKSON, M., GOLDTHORPE, J. H \& MILLS, C. (2008). Education, Employers and Class Mobility. Research in Social Stratification and Mobility, 23, 3-34.

JENCKS, C.S.,; SMITH,M.; H. ACLAND, M. J. BANE, D. COHEN, H.GINTIS, B. HEYNS, \& S. MICHELSON (1972). Inequality: A Reassessment of the Effect of Family and Schooling in America. New York: Basic Books.

LAMO DE ESPINOSA, E.(1988). Universidad y mercado de trabajo. En J. Grao (coord), Planificación de la educación y mercado de trabajo, Madrid: Narcea.

LATIESA, M. (1990). La deserción universitaria. CIS: Madrid. 
MARTÍNEZ GARCÍA, J. S.(2014). Sobrecualificación de los titulados superiores y movilidad social. Pp. 117-140 en PIAAC. Programa internacional para la evaluación de las competencias de la población adulta. 2013. Informe español. Vol II: análisis secundario. Madrid: MEC-INEE.

MINCER, J. (1974). Schooling, Experience and Earnings. New York: NBER

NAVARRO CENDEJAS, J. (2013). Universidad y mercado de trabajo en Cataluña. Un análisis de la inserción laboral de los titulados universitarios. Tesis Doctoral, Universidad Autónoma de Barcelona, 2013.

OPHEIM, V. (2007). Equal opportunities? The effect of social background on transition from education to work among graduates in Norway. Journal of Education and Work, 20 (3), 252-282

PARSONS, T. (1964). Evolutionary Universals in Society. American Sociological Review, 29 (3), 339-357.

PSACHAROPOULOS, G. (1975). Revenu et education dans les pays de l'OCDE. París: OCDE.

RODRÍGUEZ MENÉS, J. (1993). Movilidad social y cambio social en España"Revista Española de Investigaciones Sociológicas, 61, 77-126.

SEWELL, W.H. \& R.M.HAUSER (1975). Education, Ocupation and Earnings: Achievement in the Early Career. New York: Academic Press.

TORCHE, F. (2011). Is a College Degree Still the Great Equalizer? Intergenerational Mobility Across Levels of Schooling in the United States. American Journal of Sociology, 117 (3), 763-807.

TRIVENTI, M. (2013). The role of higher education stratification in the reproduction of social inequality in the labor market. Research in Social Stratification and Mobility, 32, 45-63.

USTRELL, M. (2011). La vía profesional-superior disminuye la desigualdad social en el acceso al sistema universitario. RASE 5 (1), 75-90.

\section{Correspondencia con los autores}

Julio CARABAÑA MORALES

Facultad de Educación-CFP,

C/Rector Royo-Villanova, s/n,

28040 Madrid

e-mail: carabanya@ccedu.ucm.es 